PSICOLOGIA

IBEROAMERICANA
Psicología Iberoamericana ISSN: 1405-0943

revista.psicologia@ibero.mx

Universidad Iberoamericana, Ciudad de México México

\title{
Inventario de autoatribución de adjetivos para la medición de autoconcepto y autoestima en niños mexicanos
}

Gómez-Peresmitré, Gilda; Platas Acevedo, Silvia; Pineda García, Gisela; Guzmán Saldaña, Rebeca Inventario de autoatribución de adjetivos para la medición de autoconcepto y autoestima en niños mexicanos

Psicología Iberoamericana, vol. 27, núm. 1, 2019

Universidad Iberoamericana, Ciudad de México, México

Disponible en: http://www.redalyc.org/articulo.oa?id=133960951004 
Psicología Iberoamericana, vol. 27, núm. 1,2019

Universidad Iberoamericana, Ciudad de México, México

Recepción: 02 Marzo 2019 Aprobación: 20 Mayo 2019

Redalyc: http://www.redalyc.org/ articulo.oa?id=133960951004

\section{Inventario de autoatribución de adjetivos para la medición de autoconcepto y autoestima en niños mexicanos}

\author{
Inventory of self-attribution of adjectives for the measurement \\ of self-concept and self-esteem in Mexican children \\ Gilda Gómez-Peresmitré gildag@unam.mx \\ Universidad Nacional Autónoma de México, México \\ http://orcid.org/0000-0003-1572-4012 \\ Silvia Platas Acevedo \\ Universidad National Autonomous University of Mexico, México \\ http://orcid.org/0000-0001-9878-3653 \\ Gisela Pineda García \\ Uabc Vice Rectoría Ensenada, México \\ http://orcid.org/0000-0001-9873-1589 \\ Rebeca Guzmán Saldaña \\ Universidad Autónoma del Estado de Hidalgo, México \\ D http://orcid.org/0000-0003-0877-4871
}

\begin{abstract}
Resumen: En este estudio se evalúan las propiedades psicométricas -validez/ confiabilidad- de un Inventario de Autoatribución (IAA) de adjetivos relacionados con características físicas, de personalidad y sociales, que mide autoconcepto/autoestima en niños(as) de 8-12 años. La muestra no aleatoria con $\mathrm{N}=2656$ participantes, se dividió para la aplicación de: Análisis Factoriales, Exploratorio (AFE) y Confirmatorio. Los análisis confirman la estructura arrojada por el AFE, produciendo un modelo con 31 atributos distribuidos en 5 factores teórica y empíricamente congruentes -áreas importantes del autoconcepto/autoestima y valores acordes con índices establecidos para alta bondad de ajuste-. El Análisis Discriminante mostró un 100\% de predicción/ clasificación sin error de los participantes a grupos de autoatribución positiva vs autoatribución negativa. El IAA, obtuvo valores $\alpha(.84)$ y $\omega(.92)$, con un tamaño del efecto (.84), mostrando su utilidad al cubrir necesidades de medición en importantes áreas cognoscitivas en una difícil etapa del desarrollo.
\end{abstract}

Palabras clave: propiedades psicométricas, análisis factoriales, autoatribución, autoconcepto, autoestima, niños y niñas.

Abstract: This study aimed to evaluate psychometric properties -validity/reliabilityusing the Inventory of Self-Attribution (SAI). The study measured adjectives, related to physical, personality and social characteristics, which relate to self-concept/self-esteem in children of 8-12 years. A non-random sample of 2656 participants and quantitative analysis using factor analyses, specifically Exploratory (EFA) and Confirmatory (CFA) was used. CFA confirmed the structure known by EFA and produced a model of 31 attributes distributed in five theoretically and empirically congruent factors-important areas of self-concept/self-esteem and values according to established indexes for high goodness of fit. The discriminant analysis showed a $100 \%$ prediction/classification without error of the participants to positive SA versus negative SA groups. The SAI obtained values $\alpha(.84), \omega(.92)$ and size effect (.84), showing its usefulness in measuring important cognitive areas and stages of development.

Keywords: psychometric properties, factor analysis, self-attribution, self-concept, selfesteem, children. 


\section{Introducción}

Considerando que el objetivo principal de este estudio es la aportación de un instrumento de fácil aplicación, válido y confiable para la medición del autoconcepto/autoestima en niños(as) de educación básica, deben ubicarse como un primer paso, conceptual y empíricamente, los procesos implicados en dicho propósito y definir la importancia de contribuir al desarrollo de un Auto-Concepto (AC) saludable, es decir, positivo. Los procesos implicados, tales como la percepción y la atribución, son de carácter psicosocial, coadyuvan con la formación del AC, y su funcionalidad reside en la posibilidad de proporcionar pautas para explicar la realidad, no sólo del propio comportamiento sino también del de los demás (Martínez \& González, 2017). El aprendizaje social, de modelamiento y vicario, la socialización en la familia, en la escuela, entre pares, y la de los medios de comunicación social, constituyen los principales factores formadores del AC, que como puede verse y lo señala Roa (2013), no es innato. Puede decirse que es producto del proceso evolutivo, durante el cual el niño lo va construyendo y, en la medida en la que crece hasta llegar a la adultez, va diferenciándolo cada vez más. El AC aumenta su multidimensionalidad con la edad, y en edades tempranas no existe una diferencia entre el sí mismo y el entorno, por lo que se tiene un AC global, no diferenciado y específico de cada situación (Craven \& Marsh, 2008; Zhu et al., 2016). En esa misma dirección otros investigadores, entre ellos Amezcua (2000), señalan de manera más precisa que el AC no se diferencia internamente hasta entrada la adolescencia.

El AC también contribuye a la adopción de estilos de vida y se convierte en un importante correlato del ajuste psicosocial, ya que, entre otras cosas, determina la conducta a través de las expectativas, por ejemplo, no serán las mismas respuestas si el niño se percibe positivamente que si lo hace negativamente. En este último caso, la expectativas serán negativas: el niño esperará resultados negativos en la interacción con los demás (Fuentes, García, García, \& Lila, 2011; García \& Musitu, 2001; Garrido, García, Flores, \& de Mier, 2012). De acuerdo con Garaigordobil, Pérez y Mozaz (2008) y Zhu et al. (2016), se han realizado investigaciones sobre la influencia del AC, como un indicador de satisfacción psicológica y bienestar personal en las que se muestra una correlación positiva, significativa, entre el AC y la salud mental, es decir, al facilitar el sentido de la propia identidad y la aceptación de sí mismo se contribuye a la salud y equilibrio psicológico, aspectos relevantes para lograr el desarrollo personal y social. El AC es determinante para el desarrollo de la personalidad. Un AC positivo se asocia con un desarrollo cognitivo, emocional y social saludables y es la base del buen funcionamiento personal, social y académico o profesional. Así el AC se perfila como una variable importante para el logro y mantenimiento de la salud física y psicológica, así como para la prevención de trastornos tales como la obesidad y los de la conducta alimentaria (Demaray, Rueger, Brown, \& Summers, 2009; García, Musitu, \& Veigab, 2006; Goñi \& Rodríguez, 
2004; Goñi, Ruiz de Azúa, \& Rodríguez, 2004; Pernice-Duca, 2010). De ahí que lograr un AC positivo sea y deba ser uno de los objetivos más preciados de los programas de intervención. Esta es precisamente, la intención que subyace al instrumento que en este estudio se formula. Mientras a más temprana edad se conozca o detecte la tendencia positiva/ negativa de la autoatribución, mayores serán las probabilidades de reforzar el mantenimiento/crecimiento o el cambio/re-estructuración del AC.

Las características físicas son un componente importante del AC y de la Auto-Estima (AE) que todos tenemos en cuenta en el momento de describirnos o evaluarnos. Así pues, el AC guarda una estrecha relación con la AE: si el individuo se atribuye cualidades positivas se incrementan sus autoevaluaciones con afecto positivo; si se atribuye cualidades negativas, entonces, baja la $\mathrm{AE}$ y se asocia con afecto negativo. Wickline (2003) señala, al respecto, que la $\mathrm{AE}$ y el $\mathrm{AC}$ no se pueden separar empíricamente pues como señala Fernández (2009) la distinción entre ellos no se ha demostrado ni conceptual ni empíricamente. Las definiciones han ido desde las que distinguen entre $\mathrm{AE}$ como evaluativa y AC como descriptivo (por ejemplo, Roa, 2013), hasta los que las equiparan (Bandura, 1997; Byrne, 1996; Byrne \& Shavelson, 1986; Shavelson, Hubner, \& Stanton, 1976). De hecho, la definición del AC en la que se apoya el instrumento que aquí se propone, Inventario de Autoatribución (IAA), proviene de estos últimos autores y se le considera como la percepción que el individuo tiene de sí mismo, basada en las experiencias con los demás y en las atribuciones que hace de su propia conducta.

Ahora bien, ¿dónde se ubica el IAA para la medición del AC/ $\mathrm{AE}$ en niños(as) de 8 a 12 años con respecto a los instrumentos ya existentes y cómo se justifica dicha propuesta? Probablemente uno de los instrumentos más utilizado para la medición del AC sea el que se conoce como AF5 de García y Musitu (2001). Este instrumento se ha utilizado bajo el supuesto de una composición multidimensional jerárquica del AC que se propusiera desde Shavelson et al. (1976) y, según la cual, éste se ajusta a un Modelo Pentafactorial (AF5). Su estructura interna muestra varios dominios: el académico, emocional, social, familiar y físico. Esta postura surge, en contraste con la que plantea un AC unidimensional, que subraya la totalidad, los aspectos globales e indiferenciados, por ejemplo, Marx y Winne (1978); Rosenberg (1965) entre otros. Puede decirse, en general, que los resultados reportados de las investigaciones que han utilizado el AF5, que ha sido el modelo dominante durante las últimas décadas, concuerdan con propiedades psicométricas, estadística y metodológicamente aceptables y, mientras que algunos confirman la estructura pentafactorial (Fuentes et al., 2011; García \& Musitu, 2001, 2014; García et al., 2006) otros no lo hacen (Fernández Zabala \& Goñi Palacios, 2008). Todavía más, otros autores como García, Gracia y Zelesnova (2013) afirman que una estructura de 6 factores tiene mejor fundamentación teórica, debido a que ellos encuentran que la dimensión física del AF5 se divide en atractivo físico y condición física. 
Otra línea de reciente investigación sobre atribución y AC se ha centrado en el área académica. Clem et al. (2018), Franco, Blanco, Jurado, Aguirre, y Blanco (2018), Lohbeck, Grube, y Moschner (2017), y Weiner (2004) al igual que investigadores como Inglés, MartínezGonzález, García-Fernández, Torregrosa, y Ruíz (2012) consideran que la autoatribución y el $\mathrm{AC}$ son aspectos fundamentales del componente afectivo y emocional de la motivación escolar. Jiménez (2017) encontró que existe una correlación positiva significativa entre el $\mathrm{AC}$ y el rendimiento escolar, siendo el primero un predictor del segundo. El estudio de Redondo, Inglés y García-Fernández (2014) mostró que los alumnos que se atribuían habilidades académicas positivas, tenían conductas prosociales. Ternera (2014) obtuvo correlaciones significativas entre AC negativo y bajo desarrollo de habilidades sociales.

Con respecto a la investigación nacional con el AF5 en población estudiantil, no se encontraron estudios en niños de educación básica. Se han realizado investigaciones en Monterrey, N. L. (Meza-Peña \& PompaGuajardo, 2016) con adolescentes hombres y mujeres (Medad13.17, DE .91); y en Tamaulipas, México (Salum-Fares, Marín, \& Reyes, 2011) con adolescentes hombres y mujeres (Medad14.06, DE .36). Una investigación realizada con escolares de educación primaria (7-12 años) hombres y mujeres (Gálvez Casas et al., 2015) en la que también se utilizó un instrumento multidimensional, el de Piers y Harris (1969) validado por Cardenal y Fierro (2003), mide siete dimensiones del AC, con 80 reactivos y respuesta dicotómicas sí/no $(\alpha=.89)$. En otro estudio, realizado en Sinaloa, México (Miranda, Miranda, \& Enríquez, 2011), con niños(as) de escolaridad básica, se propusieron adaptar el instrumento de Coopersmith (1967) con 58 reactivos que miden cuatro dimensiones de la AE. Se reporta una única propiedad psicométrica $(\alpha=.81)$ y se le interpreta como nivel de precisión o certeza del instrumento. Por último se encontró otro estudio, realizado en España, en el grupo de edad que aquí interesa (8-12 años) en el que se aplicó un Cuestionario de Autoconcepto Infantil con 10 reactivos. Resultaron 5 escalas: social, académica, personal, física y general. Sin embargo, obtuvo coeficientes alpha muy bajos (.46 el más alto) y los 5 factores, aunque con cargas factoriales altas $\geq 40$ quedaron, cada uno, con sólo uno o dos reactivos contrario al criterio que establece que un factor debe agrupar por lo menos tres reactivos (Hair, Anderson, Tatham, \& Black, 1998).

Queda claro que la investigación para la medición del AC en niños de educación escolar primaria es escasa y que no se cuenta (como se ha mostrado arriba) con instrumentos que cubran las necesidades técnicas y metodológicas básicas. Por otra parte, la propuesta de la multidimensionalidad del AC representa uno de los aspectos de mayor conflicto y polémica (García \& Musitu, 2014), el de la estructura interna del AC, que plantea el desarrollo evolutivo del mismo asumiendo que es hasta la adolescencia cuando éste se diferencia internamente de manera clara y la propuesta consiguiente, de la estructura interna, que va desde una perspectiva multidimensional, bidireccional a una unidireccional. Este conflicto puede verse reflejado en la pregunta que se plantean 
Fernández Zabala y Goñi Palacios (2008): ¿'Tiene sentido elaborar instrumentos de medida del autoconcepto que respondan a modelos teóricos multifacéticos?

Debe señalarse que, en este estudio, a partir de las condiciones señaladas y de las implicaciones de dicha pregunta, se decidió que fueran las respuestas de los niños quienes mostraran la estructura (multidimensional, bidimensional o unidimensional) del IAA y que éstas se vieran lo menos influidas posible por el contexto de las preguntas, por lo que se consideró utilizar, en lugar de preguntas, una lista de atributos/ adjetivos, para que los niños(as) respondieran cuánto creían tener de cada uno de ellos.

La investigación se centra en el concepto de autoatribución como un elemento esencial del AC (Lazos, 2008) y se fundamenta en las teorías de la percepción y aprendizaje social y de la socialización (Bandura, 1982, 1997; Byrne, 1996; Byrne \& Shavelson, 1986; Shavelson et al., 1976) así como en investigaciones antecedentes (Correa, 2002; Gama, 1999; Guzmán, 2002). Éstas han aportado información que ha permitido incrementar y depurar atributos o adjetivos, lo que ha favorecido que las respuestas de los niños dieran lugar a la generación de nuevas dimensiones en la estructura del constructo AC, que originalmente (González, Lizano, \& Gómez-Peresmitré, 1999) sólo eran clasificables en una o dos áreas ya fueran atributos positivos o negativos, confirmando, por otro lado, el carácter multidimensional multifacético propuesto por varios autores, como ya antes se señalara. En el presente trabajo se plantea como objetivo principal la evaluación psicométrica (validez, confiabilidad, poder predictivo y discriminante) del IAA con miras a proponerlo como una opción funcional (de fácil y rápida aplicación) para la medición ya sea del AC o de la AE en niños y niñas en la preadolescencia. La no distinción entre AC y AE se apoya en la usual práctica tradicional, como también ya antes se señalara, y también se justifica por lo relativamente transitorio de las características físicas, cognitivas y socioafectivas de la etapa etaria a la que el IAA va dirigido.

\section{Método}

\section{Participantes}

Este estudio es de carácter instrumental, de campo y transversal, de dos muestras independientes de selección no aleatoria $(\mathrm{n} 1=1341$ y n2=1315) formadas por niñas y niños de educación básica con un rango de edad entre 8 y 12 años, $M=10.9$ (DE.94).

\section{Instrumento}

El Inventario de Autoatribución (IAA) original compuesto por 41 adjetivos, positivos (22) y negativos (19), con 5 opciones de respuesta que van desde nada $(=1)$ para cuando el que responde cree no poseer nada 
del atributo en turno hasta demasiado $(=5)$ para cuando cree tener lo máximo. Los valores alpha reportados varían de .70 a .82 .

\section{Procedimiento}

Se acudió con las autoridades de escuelas públicas de educación primaria de la Ciudad de México para solicitar la participación de los alumnos de las mismas. En las juntas de los padres se solicitó a los responsables de los niños la firma del consentimiento informado para la participación de sus hijos. La aplicación se hizo de manera colectiva en los salones de clase por estudiantes de psicología, previamente entrenados, con una duración de aproximadamente 30 minutos. Previo a la aplicación del instrumento se explicó a los niños y niñas que su participación era voluntaria y que podrían no participar o no terminar de responder el cuestionario en el momento que así lo decidieran. En ninguna de las aplicaciones en las diferentes escuelas se reportaron niños o niñas que se negaran o interrumpieran el procedimiento. En todo momento se siguieron las recomendaciones éticas para procedimientos no intrusivos en investigación (SMP, 2010).

\section{Análisis de los Datos}

Se utilizó un análisis de frecuencias para determinar las distribuciones de los reactivos y probar la variabilidad y normalidad de las mismas (Muthén \& Kaplan, 1985). Asimismo, se utilizaron las pruebas de adecuación de la muestra con punto de corte $(\mathrm{KMO} \geq .60)$ y de esfericidad de Bartlett $\left(\mathrm{X}^{2} \leq .05\right)$. Se aplicó el Análisis Factorial Exploratorio (AFE) con el método de los Componentes Principales y rotación Varimax; reactivos con carga factorial ( $\geq .40)$. Se aplicó el Análisis Factorial Confirmatorio (AFC) con el método de Máxima Verosimilitud utilizando una nueva muestra. Se calcularon los valores alpha y omega ( $\geq .60)$. Se establecieron los siguientes criterios de ajuste: $\mathrm{X}^{2} / \mathrm{gl} \leq 5$; $\mathrm{AGFI} \geq .90$; CFI $\geq .90$; $\mathrm{IFI} \geq .90$; RMSEA $\leq .05$. Se aplicó un Análisis Discriminante con el Método de Mahalanobis, por pasos.

\section{Resultados}

AFE y AFC. De la muestra total de 2,656 niñas y niños se formó una muestra para la aplicación del AFE $(\mathrm{n} 1=1341)$ y otra $(\mathrm{n} 2=1315)$ para el AFC. El rango de edad en las dos muestras fue de 8 a 12 años, con una edad media de $\mathrm{M}=10.9$ (DE .94). Una vez probada la normalidad de las distribuciones de los datos (sesgo+/- 2 y curtosis $=+/ 2$ ) (Muthén $\&$ Kaplan, 1992), y probado el cumplimiento de los criterios de adecuación y el de la no esfericidad de la matriz de los datos del AFE (KMO .920; Bartlett $\mathrm{X}^{2}$ 14839.722 /820 Sig. .001); y los de los datos del AFC (KMO .920; Bartlett $\mathrm{X}^{2}$ 14560.514 /820 Sig. .001) se aplicó el AFE con el método de los Componentes Principales, rotación Varimax y el AFC 
con el Método de Máxima Verosimilitud. Se estableció como punto de corte para las cargas factoriales ( $\geq .40)$, valores alpha ( $\geq .60)$ y número de variables por factor $(\geq 3)$.

El AFE ( $1=1341)$ arrojó 6 factores (4 positivos y 2 negativos), el factor 1 , con 8 atributos positivos que exploran el área de afecto positivo (feliz, amigable, cariñosa...); factor 2, con 6 atributos positivos que exploran el área académica (estudiosa, cumplida, inteligente...); factor 3 , con 6 atributos negativos relativos al área de afecto negativo (torpe, triste, solitaria...); factor 4, con 5 atributos negativos explorando el área afectivo/conductual antisocial (mentirosa, tramposa, agresiva...); factor 5 , con 5 atributos positivos relativos al área afectivo/conductual prosocial (sociable, independiente, confiable...); factor 6 , con 3 atributos positivos midiendo el área física proactiva (fuerte, deportista, ágil...). El IAA resultante del AFE quedó formado por 33 atributos distribuidos en 6 factores (4 positivos y 2 negativos) con alpha general .85 , omega .95 y $\mathrm{S}^{2}$. total, explicada $=43.5 \%($ ver Tabla 1$)$.

\section{Tabla 1}

\begin{tabular}{|c|c|c|c|c|c|c|}
\hline \multicolumn{7}{|c|}{ Exploratorio } \\
\hline & Factor & Factor & Factor & Factor & Factor & Factor \\
\hline & 1 & 2 & 3 & 4 & 5 & 6 \\
\hline $\begin{array}{l}\text { Número } \\
\text { reactivos }\end{array}$ & 8 & 6 & 6 & 5 & 5 & 3 \\
\hline Alpha de Cronbach & .86 & .80 & .70 & .65 & .67 & .61 \\
\hline Omega & .85 & .78 & .75 & .72 & .69 & .67 \\
\hline $\begin{array}{l}\text { \% Varianza } \\
\text { Explicada }\end{array}$ & 20.01 & 9.53 & 4.31 & 3.64 & 3.16 & 283 \\
\hline Alpha general & \multicolumn{6}{|c|}{.85} \\
\hline Omega general & \multicolumn{6}{|c|}{.95} \\
\hline \multicolumn{7}{|c|}{ Confirmatorio } \\
\hline Número de ítems & 9 & 6 & 7 & 4 & 5 & $3^{*}$ \\
\hline Alpha de Cronbach & .84 & .80 & .74 & .67 & .69 & .60 \\
\hline Omega & .80 & .72 & .72 & .60 & 62 & .53 \\
\hline $\begin{array}{l}\text { \% Varianza } \\
\text { Explicada }\end{array}$ & 18.08 & 8.8 & 2.4 & 2.4 & 1.7 & 1.01 \\
\hline Alpha general & \multicolumn{6}{|c|}{.84} \\
\hline Omega general & \multicolumn{6}{|c|}{.92} \\
\hline
\end{tabular}


El AFC (n2=1315) produjo 6 factores. el factor 1, con 9 atributos positivos que exploran la misma área del AFE, es decir, afecto positivo (feliz, amigable, agradable...); factor 2, con 6 atributos positivos relacionados con el área académica (estudiosa, cumplida, inteligente...); factor 3 , con 7 atributos negativos relativos al área de afecto negativo (torpe, triste, solitaria,...); factor 4 , con 4 atributos positivos relativos al área afectivo/conductual prosocial (sociable, optimista, comprensiva...); factor 5, con 5 atributos negativos que exploran el área afectivo/ conductual antisocial (mentirosa, tramposa, agresiva...); y el factor 6 , con 3 atributos positivos midiendo el área física proactiva (fuerte, deportista y ágil). El IAA resultante del AFC quedó formado por 34 atributos distribuidos en 6 factores ( 4 positivos y 2 negativos). Con el ajuste del modelo resultante del AFC quedó eliminado el factor denominado físico proactivo compuesto por 3 atributos, quedando un total de 31 atributos, con valor general alpha y omega $\left(\alpha .84, \omega .92\right.$ y $\left.S^{2}\right)$ Tot. Explicada (34\%) (Tabla 2, Figura 1). AFE y AFC resultaron con la misma estructura y prácticamente con el mismo orden de aparición (sólo los factores 4 y 5 invirtieron su orden).

Figura 1. Modelo Factorial Confirmatorio Autoatribución.

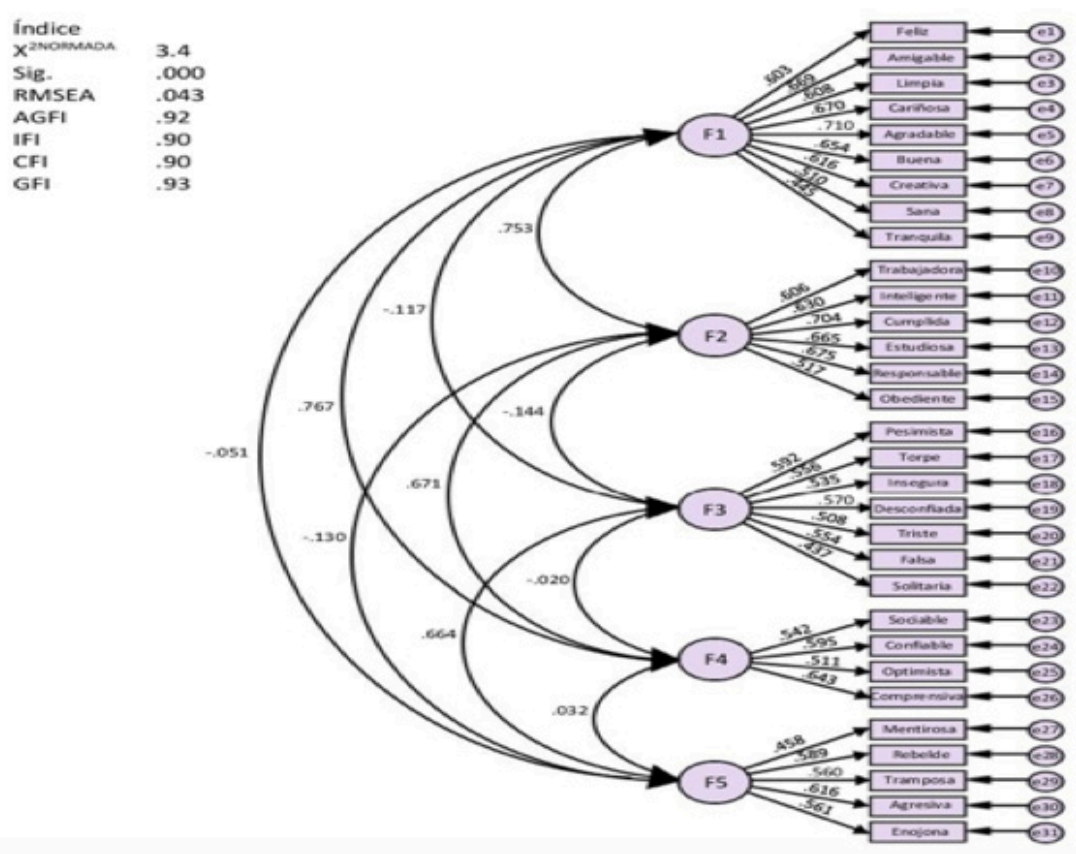

Es importante resaltar la congruencia empírico-teórica de las correlaciones (arcos con doble flecha) que se muestran en el modelo del AFC (Fig. 1). Pueden observarse correlaciones altas y positivas (afecto positivo) con habilidades académicas $(\mathrm{r}=.75)$ y con el área afectivo/ conductual prosocial $(\mathrm{r}=.76)$. Asimismo, entre habilidades académicas $\mathrm{y}$ el afectivo/conductual prosocial $(\mathrm{r}=.67)$ y las correlaciones bajas $\mathrm{y}$ negativas, por ejemplo, $\mathrm{r}=-.051$ entre afecto positivo y afectivo/ conductual antisocial. 
Análisis Discriminante (AD). Con el propósito de predecir el porcentaje de error que se cometería en la clasificación, tanto de los casos del estudio como en el de los nuevos, se utilizó el AD, aunque se comprobó que no había igualdad de matrices, como lo requiere la aplicación de éste. Sin embargo, se ha documentado que el tamaño grande de la muestra influye en la diferenciación de las matrices por lo que en esos casos dicho incumplimiento no se considera importante, además de que el AD se clasifica como una prueba robusta (Huberty, 1975, 1994). Se obtuvo un valor alto y significativo de $\mathrm{R}_{\text {can }} .917$ así como también un alto autovalor 5.29 .

En términos de varianza explicada o del tamaño del efecto se encontró $\mathrm{R}^{2} .84(84 \%)$ y $1-\mathrm{R}^{2}(\lambda 16 \%)$ de varianza no explicada. En la matriz de estructura, se agruparon 11 de los coeficientes de correlación que alcanzaron un valor $\geq .30$ (punto de corte para separar los ítems importantes de una distribución). La mayoría de éstos correspondieron a atributos del factor 1 (afecto positivo) y del factor 2 (área académica) del AFC. Por último, el AD arrojó 100\% de clasificación correcta en la validación original y 99.9\% en la validación cruzada (ver Tabla 2).

Tabla 2

Función Discriminante y Porcentajes de Clasificación Correcta (Validez Cruzada)

\begin{tabular}{|c|c|c|c|c|c|}
\hline \multirow{2}{*}{\multicolumn{2}{|c|}{$\begin{array}{r}\text { R } \\
\text { Canónica } \\
.917\end{array}$}} & Autovalor & $\begin{array}{c}\text { Varianza Explicada } \\
\mathrm{R}^{2}\end{array}$ & $\begin{array}{l}\text { Varianza No } \\
\text { Explicada } \\
1-\text { R2 }\end{array}$ & Significancia \\
\hline & & 5.29 & .84 & 人 $16 \%$ & .001 \\
\hline \multicolumn{6}{|c|}{$\begin{array}{l}\text { Grupo de pertenencia pronosticado } \\
\text { Autoatribución Positiva Autoatribución } \\
\text { Negativa }\end{array}$} \\
\hline \multirow{4}{*}{\multicolumn{2}{|c|}{$\begin{array}{l}\text { Validez } \\
\text { Original }\end{array}$}} & $\begin{array}{c}\text { Autoatribución } \\
\text { Pos. }\end{array}$ & 660 & 0 & 660 \\
\hline & & $\begin{array}{c}\text { Autoatribución } \\
\text { Neg }\end{array}$ & 0 & 665 & 665 \\
\hline & & $\begin{array}{c}\text { Casos } \\
\text { desagrupados }\end{array}$ & 711 & 620 & 1331 \\
\hline & & $\begin{array}{c}\text { Autoatribución } \\
\text { Pos. }\end{array}$ & 100,0 & .0 & 100.0 \\
\hline \multirow{2}{*}{\multicolumn{2}{|c|}{$\%$}} & $\begin{array}{c}\text { Autoatribución } \\
\text { Neg }\end{array}$ & 0 & 100,0 & 100,0 \\
\hline & & $\begin{array}{c}\text { Casos } \\
\text { desagrupados }\end{array}$ & 53,4 & 46,6 & 100,0 \\
\hline \multirow{4}{*}{$\begin{array}{l}\text { Validación } \\
\text { cruzadab }\end{array}$} & Recuento & $\begin{array}{c}\text { Autoatribución } \\
\text { Pos }\end{array}$ & 660 & 0 & 660 \\
\hline & & $\begin{array}{c}\text { Autoatribución } \\
\text { Ned }\end{array}$ & 0 & 665 & 665 \\
\hline & \multirow{2}{*}{$\%$} & $\begin{array}{l}\text { Autoatribución } \\
\text { Pos }\end{array}$ & 100,0 & 0 & 100,0 \\
\hline & & $\begin{array}{c}\text { Autoatribución } \\
\text { Neg }\end{array}$ &, 0 & 100,0 & 100,0 \\
\hline
\end{tabular}




\section{Discusión}

Determinar la validez, confiabilidad y el poder predictivo y discriminante del IAA desarrollado para medir autoconcepto/autoestima en niños fue el objetivo central de este estudio. Los resultados obtenidos fueron alentadores en general e interesantes, en especial los de las factoriales, exploratorio/confirmatorio, en tanto muestran la importancia que tiene la dimensión denominada afecto positivo. Los niños(as) se perciben con atributos descriptivos/evaluativos, por ejemplo, como personas felices (primer atributo con la carga más alta en el AFE y AFC), buenas, tranquilas, limpias, sanas, creativas, así como también con atributos favorables para la interacción social: amigables, cariñosas y agradables. Una segunda e importante dimensión fue la del área académica en donde, ocupando el primer lugar también con la carga más alta, aparece el atributo trabajadora. Pero los niños y niñas se perciben, además, como personas trabajadoras, inteligentes, cumplidas, estudiosas, responsables y obedientes. Este último, es típico de los valores más apreciados por los principales socializadores del niño(a) mexicano(a) en esa etapa del desarrollo.

Figueroa-Rodríguez, Figueroa-Sandoval, Figueroa-Rodríguez y Hernández-Rosas (2012) reportan que en su estudio ser honrado y trabajador fueron los valores más comunes. Sin embargo, este resultado es generalizable al mexicano, sean adultos o niños. En Psicología del Mexicano, Díaz Guerrero (1982), resumiendo, señala que los niños mexicanos se orientan con valores asociados al trabajo y tienden a comportarse como obedientes y afiliativos. De la misma manera, en una investigación sobre valores con niños mexicanos frente a niños franceses se encontró que trabajar es uno de los valores más importantes para los niños mexicanos. De hecho, el primer factor se denominó trabajo (Valdez-Medina, González-Arratia, Cambrón-Chi, \& Sánchez, 2008). A este panorama de autoatribución del área de afecto positivo y académica, igualmente positiva, se puede integrar la denominada afectivo/conductual prosocial con atributos también positivos y facilitadores de la interacción social en tanto pueden considerarse como algunos de los componentes de la empatía, por ejemplo, sociable, confiable, optimista y comprensiva. Con el modelo resultante del AFC (ver Figura 1) quedó eliminado un importante factor, que exploraba el área física proactiva (deportista, ágil y fuerte).

Cabe señalar que desde la perspectiva psicométrica, que es la que aquí interesa, puede entenderse fácilmente que, la pérdida del factor del área física, se debe a sus bajos valores factoriales. Ahora bien, desde una perspectiva teórico-práctica, pueden plantearse diversas razones, y una de éstas puede atribuirse a un fenómeno social actual relacionado con la inseguridad pública, que promueve que los niños tengan cada vez menos actividad física (menos posibilidades de salir a jugar y correr en el entorno físico como la calle, parque, campo deportivo, casa del amigo, etcétera.) y dediquen mayor tiempo a actividades sedentarias (las relacionadas con uso de aparatos y aplicaciones digitales, TV, etcétera.). Asimismo, los 
niños y niñas cada vez muestran menos interés por el ejercicio y en general por la actividad física (Gómez-Peresmitré, Platas Acevedo, \& Pineda Gracía; Meneses \& Ruiz, 2017; Pérez, 2009) lo que podría ser una posible consecuencia, entre otras, de las condiciones de la actividad restringida antes señalada. Por otra parte, en las escuelas públicas de educación primaria se ha venido reduciendo el tiempo dedicado a la actividad física (Bonvecchio-Arenas et al., 2009; Jennings-Aburto et al., 2009), y al mismo tiempo se ha incrementado el sobrepeso/obesidad (Malo, Castillo, \& Pajita, 2017), a tal grado que los niños mexicanos ocupan el primer lugar, a nivel internacional, en dicho problema (UNICEF, 2017). Se requiere de mayor investigación para encontrar respuestas específicas a las preguntas que surgen en este estudio con respecto a la actividad física. Por otra parte, por la pérdida de ese factor, el IAA quedó como un instrumento multidimensional (pentafactorial como el AF5), prácticamente con el mismo número de reactivos y con valores alpha iguales o más altos que los del AF5 (Fuentes et al., 2011; García \& Musitu, 2001, 2014; García et al., 2006). De la misma manera, quedó conformado por prácticamente las mismas áreas y si bien el AF5 tiene un área física, no tiene la antisocial cuya detección puede ser potencialmente importante para disminuir, antes de entrar a la adolescencia, la vulnerabilidad social que experimentan los niños y niñas, debido a la exposición directa/ indirecta, presencial/digital, etcétera, a entornos de violencia.

En el modelo resultante del AFC quedó plasmada la congruencia empírica y sentido teórico entre sus factores, como lo muestran sus correlaciones (ligas con doble flecha, ver Fig.1) que confirman los resultados de Jiménez (2017) y Redondo et al. (2014), sobre correlaciones positivas entre autoconcepto y rendimiento escolar; entre autopercepción de habilidades académicas positivas y conductas prosociales y la que señala Watson, Clark y Carey (1988) entre afecto negativo y el factor afectivo/conductual antisocial. El resto de las correlaciones (Figura 1) son prácticamente negativas o nulas, pero teóricamente congruentes. Por otro lado, el poder predictivo y clasificatorio (auto atribución positiva/ negativa) sin error del IAA de los niños(as) participantes de la muestra, resultó prácticamente inmejorable (casi del 100\%). Con igual poder predictivo/clasificatorio, pero ahora para alta vs baja AA, quedaron las áreas exploradas por cada factor, por ejemplo, afecto positivo alto/bajo, etcétera.

\section{Conclusiones}

El presente trabajo aporta los primeros resultados de un instrumento (IAA) para niños o niñas de 8 a 12 años. El IAA cuenta con 31 reactivos distribuidos en 5 factores que exploran áreas importantes componentes del autoconcepto/autoestima, tales como rasgos de personalidad o estados de ánimo, habilidades académicas y características sociales. El IAA obtuvo coeficientes de confiabilidad que superan los puntos de corte aceptables, así como los máximos porcentajes de poder predictivo y clasificatorio. Además presentaron un alto valor del tamaño del efecto ya que, como lo 
señala Cohen (1988), los valores usualmente reportados en las áreas de las ciencias sociales y de la conducta, son bajos. Es importante dejar en claro que el IAA aporta una herramienta que permite estimar tempranamente, en la pubertad o inicio de la adolescencia, si la autodescripción o autoevaluación de los niños(as) va acorde con la salud física y emocional.

\section{Limitaciones, Sugerencias y Aportaciones}

La imposibilidad de generalizar los resultados a la población de niño(as) de educación básica constituye una de las limitaciones de este estudio, por lo que se requiere realizar nuevas investigaciones para evaluar el IAA en muestras con diseños de muestreo aleatorio.

Se sugiere comprobar la estructura multidimensional (pentafactorial) así como la equivalencia psicométrica del IAA en distintas muestras.

Para responder a las preguntas del por qué se perdió el factor del área física se requiere, por ejemplo, de nuevas aplicaciones en muestras similares para determinar si debe ampliarse la gama de atributos mediante nuevos estudios de validez de contenido. También se puede comparar muestras de escuelas públicas (niños/as con actividades físicas restringidas) frente a escuelas privadas (niños/as con actividad física no restringida), equiparando el resto de variables, para determinar si la capacidad/no capacidad de disponer de áreas de juego, y de ejercicio, se relaciona o no, con la selección de atributos estadísticamente fuertes que se agrupen en una nueva dimensión en el área física.

El proceso de construcción y evaluación del IAA mostró su potencial utilidad en la medición ya sea del autoconcepto, si lo que se quiere es conocer cómo se describe así mismo el niño o la niña, o la autoestima, si lo que interesa es determinar cómo se valora y relaciona afectivamente consigo. Contribuye a este logro y propósito de medición el hecho de que los atributos se presenten como adjetivos, porque éstos forman parte del ambiente cotidiano del niño y la niña, familiarizándose con ellos desde la más temprana edad.

\section{Referencias}

Amezcua, J. A. (2000). El autoconcepto y rendimiento escolar en niños de 11 a 14 años. Granada, España: Servicio de publicaciones de la Universidad de Granada.

Bandura, A. (1982). Teoria del aprendizaje social. Madrid, España: Espasa Calpe.

Bandura, A. (1997). Self-efficacy: The exercise of control. Nueva York, NY, US: Henry Holt \& Co.

Bonvecchio-Arenas, A., Theodore, F., Hernández-Cordero, S., CampiranoNúñez, F., Islas, A., Safdie, M., \& Rivera-Dommarco, J. (2009). La escuela como alternativa en la prevención de la obesidad: La experiencia en el sistema escolar mexicano. Revista Española de Nutrición Comunitaria, 16(1), 13-16. https://doi.org/10.1016/S1135-3074(10)70005-3 
Byrne, B. M. (1996) Measuring self-concept across the lifespan: Issues and instrumentation. Washington, DC, US: American Psychological Association. http://dx.doi.org/10.1037/10197-000

Byrne, B. M., \& Shavelson, R. J. (1986). On the structure of adolescent selfconcept. Journal of Educational Psychology, 78(6), 474-481. https://doi.o $\mathrm{rg} / 10.1037 / 0022-0663.78 .6 .474$

Cardenal, V., \& Fierro, A. (2003). Componentes y correlatos del autoconcepto en la escala de Piers-Harris. Estudios de Psicología, 24(1),101-111. https:/ /doi.org/10.1174/021093903321329094

Clem, A. L., Aunola, K., Hirvonen, R., Määttä, S., Nurmi, J. E., \& Kiuru, N. (2018). Adolescents' domain-specific self-concepts of ability predict their domain-specific causal attributions: A longitudinal study. Merrill-Palmer Quarterly, 64(4), 539-569. https://doi.org/10.13110/merrpalmquar 198 2.64.4.0539

Cohen, J. (1988). Statistical power analysis for the behavioral sciences (2a ${ }^{\mathrm{a}}$. ed.). Hillsdate, NJ, US: LEA.

Coopersmith, S. (1967). The antecedents of self-esteem. Palo Alto, CA, US: Consulting Psychologists Press.

Correa, G. V. (2002). Autoatribución asociada con obesidad, sobrepeso y peso normal en una muestra de estudiantes preadolescentes del sexo masculino (Tesis de licenciatura en psicología). Universidad Nacional Autónoma de México, CDMX, México.

Craven, R. G., \& Marsh, H. W. (2008). The centrality of the self-concept construct for psychological wellbeing and unlocking human potential: Implications for child and educational psychologists. Educational and Child Psychology, 25(2), 104-118.

Demaray, M. K., Malecki, C. K., Rueger, S. Y., Brown, S. E., \& Summers, K. H. (2009). The role of youth's ratings of the importance of socially supportive behaviors in the relationship between social support and self-concept. Journal of Youth and Adolescence, 38(1), 13-28. https://doi.org/10.1007/ s10964-007-9258-3.

Díaz Guerrero, R. (1982). Psicología del mexicano. CDMX, México: Editorial Trillas.

Fernández Zabala, A., \& Goñi Palacios, E. (2008). El autoconcepto infantil: Una revisión necesaria. International Journal of Developmental and Educational Psychology, 1(2), 13-22.

Fernández, A. R. (2009). Autoconcepto físico y bienestar/malestar psicológico en la adolescencia. Revista de Psicodidáctica, 14(1), 155-158.

Figueroa-Rodríguez, K. A., Figueroa-Sandoval, B., Figueroa-Rodríguez, B., \& Hernández-Rosas, F. (2012). Análisis de los valores que construyen la identidad del mexicano. Culturales, 8(16), 7-32.

Franco, D. H., Blanco, V. H., Jurado, G. P. J., Aguirre, V. I., \& Blanco, O. R. (2018). Invarianza factorial del cuestionario de autoconcepto dimensional audim-m en hombres y mujeres universitarios mexicanos. European Scientific Journal, 14(29), 231-243. http://dx.doi.org/10.1904 4/esj.2018.v14n29p231

Fuentes, M., García, J., García, E., \& Lila, M. (2011). Autoconcepto y ajuste psicosocial en la adolescencia. Psicothema, 23(1), 7-12. 
Gálvez Casas, A., Rodríguez García, P. L., Rosa-Guillamón, A., García-Cantó, E., Pérez-Soto, J. J., Tarraga- Marcos, L., \& Tarraga- López, P. (2015). Relación entre el estatus de peso corporal y el autoconcepto en escolares. Nutrición Hospitalaria, 31(2), 730-736. http://dx.doi.org/10.3305/nh.2 015.31.2.8467

Gama, A. E. (1999). Factores de riesgo para los trastornos de la alimentación: Relación entre obesidad y las variables autoatribución, conductas alimentarias e imagen corporal (Tesis de licenciatura en psicología). Universidad Nacional Autónoma de México, CDMX, México.

Garaigordobil, M., Pérez, J. I., \& Mozaz, M. (2008). Self-concept, self-esteem and psychopathological symptoms. Psicothema, 20(1), 114-123.

García, J. F., \& Musitu, G. (2001). AF5. Autoconcepto forma 5. Madrid, España: TEA

García, J. F., \& Musitu, G. (2014) AF5: Autoconcepto forma 5 (4ª ed.). Madrid, España: TEA.

García, J. F., Gracia, E., \& Zelesnova, A. (2013). Validation of the English version of the five-factor self-concept questionnaire. Psicothema, 25(4), 549-555. https://doi.org/10.7334/psicothema2013.33

García, J. F., Musitu, G., \& Veiga, F. (2006). Autoconcepto en adultos de España y Portugal. Psicothema, 18(3), 551-556.

Garrido, R. E. R., García, A. V., Flores, J. L. P., \& de Mier, R. J. R. (2012). Actividad físico deportiva, autoconcepto físico y bienestar psicológico en la adolescencia. Retos: Nuevas Tendencias en Educación Física, Deporte y Recreación, (22), 19-23.

Gómez-Peresmitré, G., Platas Acevedo, S., \& Pineda Gracía, G. (2018). Programa de autoeficacia hacia hábitos saludables para la prevención de la obesidad en escolares mexicanos. Revista de Psicología Clínica en Niños y Adolescentes, 6(1), 44-50. https://doi.org/10.21134/rpcna.2019.06.1.6

González, L., Lizano, M., \& Gómez-Peresmitré, G. (1999). Factores de riesgo en desórdenes del comer: Hábitos alimentarios y autoatribución en una muestra de niños escolares mexicanos. Revista Mexicana de Psicología, 16(1), 117-126.

Goñi, A., \& Rodríguez, A. (2004). Trastornos de la conducta alimentaria, práctica deportiva y autoconcepto físico en adolescentes. Actas Españolas de Psiquiatría, 32(1), 29-36.

Goñi, A., Ruiz de Azúa, S., \& Rodríguez, A. (2004) Deporte y autoconcepto físico en la preadolescencia. APUNTS: Educación Física y Deportes, 3(77), $18-24$

Guzmán, U. L. A. (2002). Factores de riesgo asociados con imagen corporal, autoestima y autoatribución en preadolescentes (Tesis de licenciatura en psicología). Universidad Nacional Autónoma de México, CDMX, México.

Hair, J. E., Anderson, R. E., Tatham, R. L., \& Black W. C. (1998). Multivariate data analysis ( $5^{\mathrm{a}}$. ed.). Upper Saddle River, NJ, US: Prentice-Hall.

Huberty, C. (1975). Discriminant analysis. Review of Educational Research, 45(4), 543-598. https://doi.org/10.3102\%2F00346543045004543

Huberty, C. (1994). Applied discriminant analysis. Nueva York, NY, US: John Wiley \& Sons. 
Inglés, C., Martínez-González, A., García-Fernández, J., Torregrosa, M., \& Ruíz, C. (2012). La conducta psicosocial y el autoconcepto de estudiantes españoles de Educación Secundaria Obligatoria. Revista de Psicodidáctica, 17(1), 135-156.

Jennings-Aburto, N., Nava, F., Bonvecchio, A., Safdie, M., González-Casanova, I., Gust, T., \& Rivera, J. (2009). Physical activity during the school day in public primary schools in Mexico City. Salud Pública de México, 51(2), 141-147.

Jiménez, C. (2017). Ansiedad escolar y su relación con las autoatribuciones académicas, el autoconcepto, perfeccionismo y agresividad en una muestra de estudiantes ecuatorianos de 12 a 18 años (Tesis doctoral). Universidad de Alicante, España.

Lazos, E. (2008). Autoconocimiento: Una idea tensa.Diánoia, 53(61), 169-188.

Lohbeck, A., Grube, D., \& Moschner, B. (2017). Academic self-concept and causal attributions for success and failure amongst elementary school children. International Journal of Early Years Education, 25(2), 190-203. https://doi.org/10.1080/09669760.2017.1301806

Malo, S. M., Castillo, N., \& Pajita, D. (2017). La obesidad en el mundo. Anales de la Facultad de Medicina, 78(2),173-178. http://dx.doi.org/10.15381/ anales.v78i2.13213

Martínez, F. D., \& González, J. (2017). Autoconcepto, práctica de actividad física y respuesta social en adolescentes: Relaciones con el rendimiento académico. Revista Iberoamericana de Educación, 73(1), 87-108.

Marx, R. W., \& Winne, P. H. (1978). Construct interpretations of three self-concept inventories. American Educational Research Journal, 15(1), 99-109. https://doi.org/10.3102\%2F00028312015001099

Meneses, M. M. M., \& Ruiz, J. F. R. (2017). Estudio longitudinal de los comportamientos y el nivel de actividad físico-deportiva en el tiempo libre en estudiantes de Costa Rica, México y España. Retos: Nuevas Tendencias en Educación Física, Deporte y Recreación, (31), 219-226.

Meza-Peña, C., \& Pompa-Guajardo, E. (2016). Género, obesidad y autoconcepto en una muestra de adolescentes de México. RICYDE: Revista Internacional de Ciencias del Deporte, XII(44), 137-148.

Miranda, J. B., Miranda, J. F., \& Enríquez, A. (2011). Adaptación del Inventario de Autoestima Coopersmith para alumnos mexicanos de educación primaria. Praxis Investigativa ReDIE: Revista Electrónica de la Red Durango de Investigadores Educativos, 3(4), 5-14.

Muthén, B., \& Kaplan, D. (1985). A comparison of some methodologies for the factor analysis of non\#normal Likert variables. British Journal of Mathematical and Statistical Psychology, 38(2), 171-189. https://doi.org/ 10.1111/j.2044-8317.1985.tb00832.x

Pérez, B. A. M. (2009). Impacto de la clase de educación física sobre la actividad moderada y vigorosa en niños de primaria. Revista Mexicana de Investigación en Cultura Fisica y Deporte, 1(1) 150-173.

Pernice\#Duca, F. (2010). Family network support and mental health recovery. Journal of Marital and Family Therapy, 36(1), 13-27. https://doi.org/10. $1111 /$ j.1752-0606.2009.00182.x

Piers, E. V. (1969). Piers-Harris children's self-concept scale. Nashville, TN, US: Counselor Recordings and Tests. 
Redondo, J., Ingles, C. J., \& García-Fernández, J. M. (2014). Conducta prosocial y autoatribuciones académicas en Educación Secundaria Obligatoria. Anales de Psicología, 30(2), 482-489. https://doi.org/10.6018/analesps.3 0.2.148331

Roa, G. A. (2013). La educación emocional, el autoconcepto, la autoestima y su importancia en la infancia. Revista Edetania, (44), 241-257.

Rosenberg, M. (1965). Society and the adolescent self-image. Princeton, NJ, US: Princeton University Press.

Salum-Fares, A., \& Marín A. R., \& Reyes, A. C. (2011). Autoconcepto y rendimiento académico en estudiantes de escuelas secundarias públicas y privadas de Ciudad Victoria, Tamaulipas, México. Revista Internacional de Ciencias Sociales y Humanidades, SOCIOTAM, XXI(1), 207-229

Shavelson, R. J., Hubner, J. J., \& Stanton, G. C. (1976). Self-concept: Validation of construct interpretations. Review of Educational Research, 46(3), 407-441. https://doi.org/10.3102\%2F00346543046003407

Sociedad Mexicana de Psicología [SMP]. (2010). Código ético del psicólogo. CDMX, México: Trillas.

Ternera, L. A. C. (2014). El desarrollo del autoconcepto en niños y niñas y su relación con la interacción social en la infancia. Psicogente, 17(31), 67-69. https://doi.org/10.17081/psico.17.31.1470

UNICEF (2017). México: Salud y nutrición. Recuperado de https://www.unic ef.org/mexico/salud-y-nutrici\%C3\%B3n

Valdez-Medina, J., González-Arratia, L. N., Cambrón-Chi, C., \& Sánchez, V.Z. (2008). Los valores en niños mexicanos y franceses. CIENCIA Ergo-Sum, 15(2), 133-138.

Watson, D., Clark, L. A., \& Carey, G. (1988). Positive and negative affectivity and their relation to anxiety and depressive disorders. Journal of Abnormal Psychology, 97(3), 346-353. http://dx.doi.org/10.1037/0021-843X.97.3. 346

Weiner, B. (2004). Attribution theory revised: Transforming cultural plurality onto theoretical unity. En D. M. McInerney \& S. V. Etten (Eds.), Big theories revisited (pp. 13-29). Greenwich, CT, US: Information Age Publishing.

Wickline, V. (2003). Ethnic differences in the self-esteem/ academic achievement relationship: A meta-analysis. Trabajo presentado en la Annual Conference of the American Psychological Association, Toronto, Canadá.

Zhu, J., Wang, X., Liu, Z., Liu, T., Wei, G., \& Chen, X. (2016). The relationship between self-concept and mental health among Chinese college students: The mediating effect of social adjusting. Open Journal of Social Sciences, 4(12), 118-125. https://doi.org/10.4236/jss.2016.412011

\section{Notas de autor}

$$
\text { gildag@unam.mx }
$$

